groups (control versus experimental) before the use of oxybutynin showed the following values of $\mathrm{p}$ : $\mathrm{Fc}-0.007 ; \mathrm{Ci}-0.0002$; Tf 0.768 ; Tc $-0.492 ; \mathrm{Vc}-0.055$. After the use of oxybutynin the corresponding values were: $\mathrm{Fc}-\mathrm{p}=0.055 ; \mathrm{Ci}-\mathrm{p}=0.0002 ; \mathrm{Tf}-0.957$; $\mathrm{Tc}-\mathrm{p}=0.181 ; \mathrm{V} c-\mathrm{p}=0.206$. Conclusion: The oxybutynin chloride was able to control bladder overactivity expressed by a significant reduction in frequency, but no changes in other parameters.

KEY WORDS: Cystitis, frequency. Oxybutynin. Cyclophosphamide.

\title{
CORRESPONDÊNCIA:
}

Haylton Jorge Suaid

Hospital das Clínicas - Departamento de Cirurgia

Av. Bandeirantes, $3900-9^{\circ}$ Andar

Ribeirão Preto, CEP: 14048-900

10 - ARTIGO ORIGINAL

\section{Ação do citrato de sildenafil sobre a função uretral de ratas com desnervação vesical ${ }^{1}$}

\author{
Haylton Jorge Suaid ${ }^{2}$, Adauto José Cologna ${ }^{3}$, Antonio Carlos Pereira Martins ${ }^{4}$, Silvio Tucci Jr
} Antonio Antunes Rodrigues ${ }^{5}$, José Anastácio Dias Neto ${ }^{6}$

\begin{abstract}
Suaid HJ, Cologna AJ, Martins ACP, Tucci Jr S, Rodrigues AA, Dias Neto JA. Ação do citrato de sildenafil sobre a função uretral de ratas com desnervação vesical. Acta Cir Bras [serial online] 2003 vol 18 suppl 5. Disponível em www.scielo.br/acb.

RESUMO - O óxido nítrico atua como neurotransmissor não adrenérgico e não colinérgico na bexiga e na uretra. Sua forma de ação se faz pela ativação da guanilatociclase responsável pela transformação de GMP em GMPc que promove o relaxamento da fibra muscular lisa. $\mathrm{O}$ citrato de sildenafil causa aumento do GMPc, através da inibição de fosfodiesterases, que hidrolisam o GMPc. Assim, o objetivo do experimento foi verificar sua ação na uretra. Métodos: Estudou-se 6 ratas com peso aproximado de $200 \mathrm{~g}$. A anestesia foi com uretana na dose de $1,25 \mathrm{mg} / \mathrm{kg}$. As cistometrias, em número de 3, foram realizadas através de cistostomia com cateter P50. A primeira logo após a cistostomia; a segunda depois da desnervação cirúrgica da bexiga e a terceira uma hora após a infusão gástrica do citrato de sildenafil. $\mathrm{O}$ sistema de registro das pressões constou de uma bomba de infusão contínua regulada para $0,3 \mathrm{ml} /$ minuto conectada em Y com o cateter P50 e a um polígrafo Narco-Bioystem. Nas cistometrias avaliou-se as pressões vesicais máxima e mínima nos momentos: normais-(I); desnervadas (II) e desnervadas com citrato de sildenafil na dose de $1 \mathrm{mg} / \mathrm{kg}$ (III). A análise estatística foi feita pelo método de Wilcoxon. Resultados: As médias das pressões máximas (pma) foram: momentos I ( $\mathrm{x}=86,6-\mathrm{SD}=10,1)$; momentos II $(\mathrm{x}=42,6-\mathrm{SD}=15,0)$ e momentos III ( $\mathrm{x}=30,8-\mathrm{SD}=12,4)$. As médias das pressões mínimas (pmi) foram: momentos I $(\mathrm{x}=72,1-\mathrm{SD}=18,9)$; momentos II $(\mathrm{x}=31,1-\mathrm{SD}=9,8)$; momentos III $(\mathrm{x}=14,5-\mathrm{SD}=9,5)$. A análise estatística entre as pma e pmi no mesmo grupo mostrou $\mathrm{p}<0,01$ para o grupo III, sendo maior que 0,05 para os demais. A comparação das pma revelou p< 0,002 entre os momentos I-II e I-III, sendo maior que 0,05 entre os momentos II e III. A comparação das pmi mostrou p< 0,004 entre os momentos I-II; $\mathrm{p}<0,002$ entre os momentos I -III e p <0,01 entre os momentos II-III, havendo portanto nível de significância entre os 3 momentos. Conclusões: 1) A desnervação vesical promove queda nas pressões uretrais máxima e mínima; 2) O Citrato de sildenafil amplia a faixa pressórica de trabalho da uretra devido a diminuição da pressão mínima; 3) O citrato de sildenafil não altera a pressão uretral máxima depois da desnervação.

DESCRITORES: Uretra. Oxido Nítrico. Citrato de Sildenafil.
\end{abstract}

\section{INTRODUÇÃo}

O óxido nítrico está sendo considerado um neurotransmissor não adrenérgico - não colinérgico, da inervação aferente de alguns órgãos e dentre eles encontra-se a bexiga, uretra e penis ${ }^{1,2}$. $\mathrm{Na}$ uretra sua ação tem sido estudada tanto na musculatura lisa quanto na estriada. Assim, encontrou-se que a via NO tem importante papel no relaxamento do esfincter interno durante as contrações vesicais rítmicas ${ }^{3}$. Estudos imunohistoquímicos mostraram maior atividade da NOS e NADPH na uretra proximal, sendo também encontrada a presença de nervos nitrinérgicos e NOS no sarcolema de fibras estriadas do esfincter externo ${ }^{4}$. A via de atuação do NO se faz pela ativação da guanilase ciclase que transforma o GMP em GMPc, que atua como miorrelaxante. A hidrolise do GMPc é feita por uma série de enzimas chamadas fosfodiesterases. O citrato de Sildenafil é um bloqueador da fosfodiesterase 5, que promove o aumento do teor de $\mathrm{GMPc}^{5}$. Na uretra pré-contraída de camundongos provoca aumento do tempo de relaxamento e da amplitude do estímulo elétrico ${ }^{6}$ "in vitro".

\section{OBJETIVO}

Em vista aos achados acima descritos, resolveuse verificar a ação do citrato de sildenafil sobre a função uretral de ratas.

\section{MÉTODOS}

1) ANIMAIS

Usou-se 6 ratas da raça Wistar, com peso aproximado de 200 gramas.

\section{2) ANESTESIA}

As ratas foram anestesiadas com uretana na dosagem de 1,250 $\mathrm{mg}$ por kilo de peso, via intraperitonial.

\section{3) PROCEDIMENTOS}

a) Cistostomia: Inicialmente foi feita tricotomia abdominal. Depois a laparotomia com exposição,

1. Trabalho realizado no setor de Cirurgia Experimental do Departamento de Cirurgia e Anatomia - HCFMRP-USP

2. Prof. Associado do Depto de Cirurgia e Anatomia - Disciplina de Urologia da FMRP-USP

3. Prof. Doutor do Depto de Cirurgia e Anatomia - Disciplina de Urologia da FMRP-USP

4. Prof. Titular do Depto de Cirurgia e anatomia - Disciplina de Urologia da FMRP-USP

5. Pós-Graduando do Depto de cirurgia e anatomia da FMRP - USP

6. Médico Residente da Disc. De Urologia do Depto de Cirurgia e Anatomia da FMRP-USP 
reparo e abertura do fundo vesical, local onde se introduziu e fixou um cateter de polietileno PE50. Esse cateter foi exteriorizado por contra abertura na região dorso lateral direita onde também foi fixada. Serviu para se fazer as medidas das pressões vesicais.

b) Desnervação vesical: Após ter sido feito o registro da pressão vesical das ratas normais, procedeu-se a desnervação vesical em todas as ratas. Constou de dissecção de toda a bexiga até o nível de transição do colo vesical com a uretra, inclusive com a secção de ambos os ureteres.

\section{4) REGISTRO DAS PRESSÕES VESICAIS}

O sistema para se fazer o registro das pressões veisicais foi composto por uma bomba de infusão continua regula para um fluxo de $0,3 \mathrm{ml} / \mathrm{minuto}$, conectada em Y ao cateter de cistostomia e a um poligrafo Narco-Biosystem. Determinou-se assim, as alterações das pressões vesicais durante todo o período de enchimento e esvaziamento vesical. As pressões foram registradas após a cistostomia ( fig 1 ), após a desnervação vesical (fig 2) e uma hora após a infusão gátrica do citrato de sildenafil na dosagem de $1 \mathrm{mg} / \mathrm{kg}$ de peso ( fig 3).

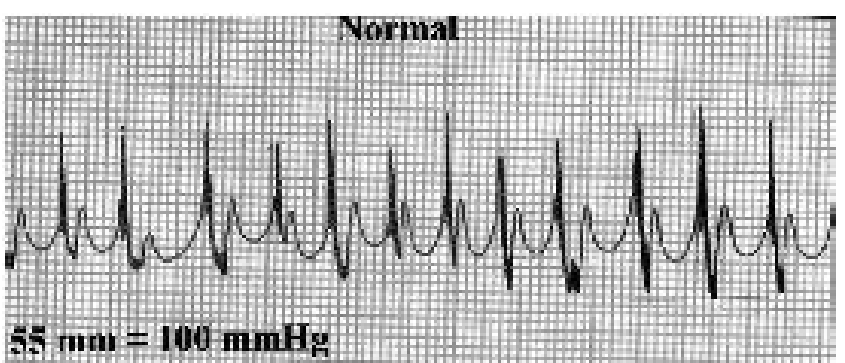

FIGURA 1 - Registro da cistomanometria de rata normal.

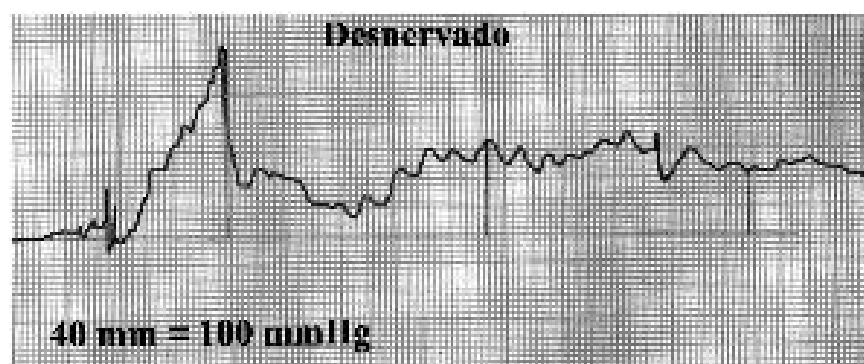

FIGURA 2 - Registro da cistomanometria de rata com bexiga desnervada

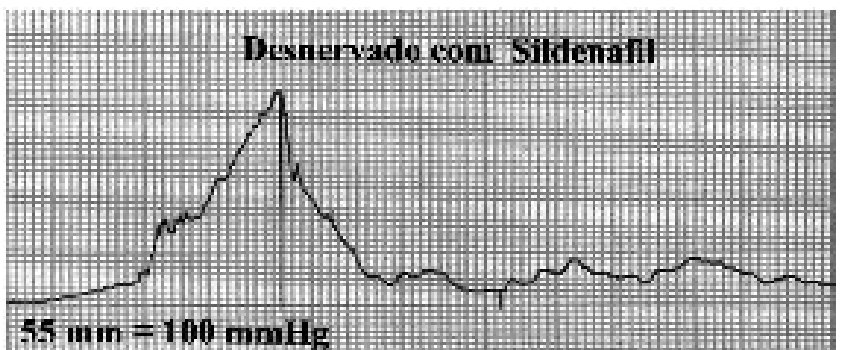

FIGURA 3 - Registro da cistomanometria de rata com desnervação vesical e citrato de sildenafil.

\section{5) LEITURA DOS REGISTROS}

Durante o período de observação foram identificadas e anotadas as pressões vesicais máximas e mínimas. Os valores pressóricos foram extraídos da relação de calibração pressão espaço demarcado.

\section{6) ANÁLISE ESTATÍSTICA}

Os resultados das pressões vesicais das 6 ratas nos três momentos estudados foram analisadas e comparadas através do método de Wilcoxon.

TABELA 1 - valores da pressões vesicais máximas e mínimas das 6 ratas estudadas.

\begin{tabular}{c|c|c|c|c|c|c}
\hline RATAS & PMa-N & PMI-N & PMa-D & PMi-D & PMa-CS & PMI-CS \\
\hline 1 & 90 & 90 & 72 & 47 & 55 & 31 \\
\hline 2 & 94 & 89 & 40 & 33 & 23 & 15 \\
\hline 3 & 100 & 81 & 28 & 25 & 23 & 15 \\
\hline 4 & 86 & 72 & 33 & 18 & 33 & 5 \\
\hline 5 & 76 & 41 & 85 & 28 & 72 & 5 \\
\hline 6 & 74 & 60 & 39 & 35 & 38 & 16 \\
\hline MEDIA & 86,5 & 72,1 & 42,6 & 31,1 & 30,8 & 14,5 \\
\hline STD & 10,7 & 18,9 & 15,0 & 9,8 & 12,4 & 9,5 \\
\hline
\end{tabular}

TABELA 2. Análise estatística das pressões vesicais máximas e mínimas dos três momentos estudados.

\begin{tabular}{ccc|c}
\hline \multicolumn{2}{c|}{ PRESSÕES VESICAIS } & VALOR DE P \\
\hline PMa-N & X & PMi $-\mathrm{N}$ & 0,132 \\
\hline PMa-N & X & PMa-D & 0,0022 \\
\hline PMa-N & X & PMa-CS & 0,0022 \\
\hline PMa-D & X & PMi-D & 0,1797 \\
\hline PMa-D & X & PMa-CS & 0,132 \\
\hline PMa-CS & X & PMi-CS & 0,0152 \\
\hline PMi-N & X & PMi-D & 0,0043 \\
\hline PMi-N & X & PMi-CS & 0,0022 \\
\hline PMi-D & X & PMi-CS & 0,0152
\end{tabular}

Legenda: Pma-N=pressão máxima de bexiga normal - PMI-N=] pressão mínima de rata normal - Pma-D=pressão máxima de bexiga desnervada

PMI-D = pressão mínima de bexiga desnervada PMa-CS= Pressão máxima de bexiga desnervada com citrato de sildenafil - PMI-CS=Pressão mínima de bexiga desnervada com citrato de sildenafil.

Legenda: Pma-N=pressão máxima de bexiga normal - PMI-N=] pressão mínima de rata normal - Pma-D=pressão máxima de bexiga desnervada

PMI-D= pressão mínima de bexiga desnervada PMa-CS= Pressão máxima de bexiga desnervada com citrato de sildenafil - PMI-CS=Pressão mínima de bexiga desnervada com citrato de sildenafil. 


\section{DISCUSSÃO}

No presente trabalho, realizou-se o bloqueio da fosfodiesterase pelo citrato de sildenafil em ratas com a bexiga desnervada. os resultados mostraram que os picos pressóricos vesicais máximos e minimos para micção não variam nas ratas normais e nem naquelas desnervadas, ocorrendo variação significante após a infusão gástrica do citrato de sildenafil. Observou-se queda significante das pressões máximas e mínimas quando se compara as ratas normais com as desnervadas sem e com citrato de sildenafil. A comparação entre as pressões máximas das bexigas desnervadas e com o citrato de sildenafil mostrou que não há nível de significância, mas quando se compara as pressões mínimas observa-se que houve queda significante. A interpretação do modelo experimental mostra que após a desnervação a bexiga se comporta como um reservatório elástico ${ }^{8}$, cuja pressão interna depende do fluxo de vazão, uma vez que o fluxo de infusão vesical é contínuo. Essa pressão é proporcional à resistência da uretra $\left(\mathrm{R}=\mathrm{P} / \mathrm{Q}^{2}\right)^{6}$, que por sua vez traduz a pressão de fechamento uretral. Assim, as pressões vesicais das bexigas desnervadas e com o citrato de sildenafil expressam as pressões de fechamento da uretra nas condições do experimento. É interessante a observação de que as pressões máximas das bexigas desnervadas sem e com o citrato de sildenafil são semelhantes , mas diferem em relação às pressões mínimas. Esse fato pode espelhar o que ocorre com o corpo cavernoso em que há necessidade do estímulo inicial para que ocorra a ação relaxadora da droga. Esses achados necessitam de estudos mais profundos para sob o ponto de vista farmacológico verificar se essa droga terá aplicação clínica em situações que haja necessidade de diminuição da pressão mínima da uretra.

\section{CONCLUSÕES}

1) A desnervação vesical promove queda nas pressões uretrais máxima e mínima;

2) O citrato de sildenafil amplia a faixa pressórica de trabalho da uretra devido a diminuição da pressão mínima;

3) O citrato de sildenafil não altera a pressão uretral máxima depois da desnervação.

\section{REFERÊNCIAS}

1. Anderson KE e Persson K. The L-arginine/ nitric oxide pathway and non-adrenergic, non-cholinergic relaxation of the lower urinary tract. Gen Pharmacol 1993; 24(4): 833-9.

2. Hoyle $\mathrm{CH}$. Non-adrenergic, non cholinergic control of the urinary bladder. World J Urol 1994; 12(5): 233-44.
3. Kawahara T. Experimental studies on effect on nitric oxide on the internal uretral relaxation in anesthetized rats. Nippon Hinyokika Zasshi - 1994,85: 1124-30

4. Takeda M, Lepor H. Nitric oxide synthase in dog uretra: a histochemical and pharmacological analysis. Br J Pharmacol 1995; 116: 2517-23.

5. Naylor AM, Ballard AS, Gingell CJC, Tang K, Turner LA. Sildenafil: an inhibitor of cyclic GMP specific phosphodiestese type 5 for the treatment of male erectile dysfunction. Eur Urol 1996; 30(suppl 2): 158. Abstract.

6. Fujiwara,m, Anderson Karl-Erik, Persson K. Nitric oxide-induced cGMP accumulation in the mouse bladder is not related to smooth muscle relaxation. Eur. J Pharmacol 2000; 401: 241-250

7. Zinner, NR. Clinical urodynamics: III Pressure profiles of the female uretrha. Surg Forum Clin Cong 1963; 14: 181.

8. Suaid HJ, Martins ACP, Cologna AJ, Tucci Jr. S; Nina Rocha, J. Comparação da funhção uretral após cistectomia simples em ratas e cistoprostatectomia em ratos. Acta Cir Bras $1998 ; 13: 25$.

\begin{abstract}
Suaid HJ, Cologna AJ, Martins ACP, Tucci Jr S, Rodrigues AA, Dias Neto JA. Effect of sildenafil citrate in the urethral function in rats with denervated bladder. Acta Cir Bras [serial online] 2003 vol 18 suppl 5. Available in www.scielo.br/acb.

ABSTRACT - Background: Nitric oxide acts as a non-adrenergic and non-cholinergic neurotransmitter in the bladder and urethra. It activates the guanilatocyclase that transforms GMP in cGMP which promotes muscle relaxation. Sildenafil citrate increases the cGMP concentration by inhibiting the phosphodiesterase responsible for its hydrolysis. Methods: 6 female rats weighing $200 \mathrm{~g}$ were anesthetized with urethane at a dosage of $1.25 \mathrm{mg} / \mathrm{kg}$. All animals underwent cystostomy with a catheter P50 connected by a Y to an infusion pump and to a polygraph Narco-Biosystem. The cystometry was performed trice in each animal: right after the cystostomy, after surgical of bladder denervation and $1 \mathrm{~h}$ after gastric infusion of $1 \mathrm{mg} / \mathrm{kg}$ of sildenafil citrate. Maximum (MaP) and minimum (MiP) vesical pressure were compared in the following moments: I - before bladder denervation, II - after bladder denervation and III - after bladder denervation and sildenafil administration. Wilcoxon test was used for a level of significance of 5\%. Results: Mean values of MaP were: I $-86.6 \pm 10.1$, II $-42.6 \pm 15.0$ and III $-30.8 \pm 12.4$. The corresponding values of MiP were: : I $-72.1 \pm 18.9$, II $-31.1 \pm 9.8$ and III $-14.5 \pm 9.5$. The comparison between $\mathrm{MaP}$ and MiP in each moment showed difference only in moment III (p<0.01). For MaP $\mathrm{p}$ value was $<0.002$ in IxII and IxIII and $>0.05$ in IIxIII. For MiP the p values were $<0.004$ in IxII, $<0.002$ in IxIII and $<0.01$ in IIxIII. Conclusion: 1) Bladder denervation reduces maximum and minimum urethral pressure; 2) Sildenafil citrate reduces the minimum urethral pressure widening the interval between the peak and bottom pressures; and, 3) Sildenafil citrate does not interfere on the peak urethral pressure after bladder denervation.
\end{abstract}

KEY WORDS: Sildenafil citrate. Denervation. Urethra. Bladder. Urodynamics.

\title{
CORRESPONDÊNCIA:
}

Haylton Jorge Suaid

Hospital das Clínicas - Departamento de Cirurgia

Av. Bandeirantes, $3900-9^{\circ}$ Andar

Ribeirão Preto, CEP: 14048-900

11 - ARTIGO ORIGINAL

\section{Reactive oxygen species inactivation improves pancreatic capillary blood flow in caerulein-induced pancreatitis in rats}

\author{
Roberto Ferreira Meirelles Jr. ${ }^{1}$, Reginaldo Ceneviva ${ }^{2}$, José Liberato Ferreira Caboclo ${ }^{3}$, Michael M. Eisenberg ${ }^{4}$
}

Meirelles RFJr, Ceneviva R, Caboclo JLF, Eisenberg MM. Reactive oxygen species inactivation improves pancreatic capillary blood flow in caerulein-induced pancreatitis in rats. Acta Cir Bras [serial online] 2003 vol 18 suppl 5. Available in www.scielo.br/acb. 